\title{
Seguridad alimentaria y diversidad dietética en el hogar, reporte de estudio en madres oaxaqueñas durante lactancia materna complementaria a bebés de 6 a 24 meses de edad
}

\author{
Food security and dietary diversity at home, study report in Oaxacan mothers \\ during complementary breastfeeding in babies from 6 to 24 months of age \\ Xunaxi Magdalena Osorio Álvarez ${ }^{a}$, Edú Ortega Ibarra ${ }^{b}$, Ilse Haide Ortega Ibarra ${ }^{c}$
}

\begin{abstract}
:
Introduction: The nutrition of mothers and their children is one of the most effective tools to achieve the optimal growth and development of the human being. Objective: To know the level of food and nutritional security of mothers who provided complementary breastfeeding (CML) to babies from 6 to 24 months of age. Method: descriptive, transversal and retrospective design. 46 mothers from Magdalena Tlacotepec and Ixtepec city, Oaxaca. They were interviewed using surveys of the Mexican Food Safety Scale (E.M.S.A.), sociodemographic, Introduction of Complementary Feeding and Dietary Diversity Questionnaire in the Home. Microsoft Office Excel 2013 spreadsheets. Results: Maternal age of 26 years, mostly from Magdalena Tlacotepec with children on average of 17.32 months. $96 \%$ had Food Security. The mothers were married, with 2 or more children, $87 \%$ lived with 4 or more people at home. $91 \%$ of the mothers interviewed lived in their own house, $98 \%$ in the house of earthenware. $48 \%$ had household appliances. In $93 \%$ of households 2 people received a salary, mostly working full-time. In 56\% of cases, 4 or more people depended on family income. Fashion school was preparatory. $67 \%$ performed housework, $52 \%$ had Seguro Popular. The infusions were introduced in the third month as the first drink different from breast milk. $98 \%$ had a high food diversity, the group with the highest consumption in the family home was cereals. Conclusions: Sociodemographic observation of 46 mothers who mostly worked in the home, had their own house of concrete, 2 or more children and with popular insurance. The people interviewed had Food Safety, an average for starting complementary feeding at 3 months with homemade infusions and families had High Alimentary Diversity.
\end{abstract}

Keywords:

Breastfeeding, Feeding Complement, Food Security, Food diversity

Resumen:

Introducción: La nutrición de las madres y sus hijos es una de las herramientas más efectivas para lograr el óptimo crecimiento y desarrollo del ser humano. Objetivo: Conocer el Nivel de Seguridad Alimentaria y Nutricional de las madres que brindaban Lactancia Materna Complementaria (LMC) a bebés de 6 a 24 meses de edad. Método: Diseño descriptivo, transversal y retrospectivo, 46 madres de Magdalena Tlacotepec y Ciudad Ixtepec, Oaxaca. Entrevistadas empleando encuestas de la Escala Mexicana de Seguridad Alimentaria (E.M.S.A.), sociodemográfica, de Introducción de Alimentación Complementaria y Cuestionario de Diversidad Dietética en el Hogar. Hojas de cálculo de Microsoft Office Excel 2013. Resultados: El 96\% tenía Seguridad Alimentaria. Moda de edad materna de 26 años, mayormente de Magdalena Tlacotepec con hijos en promedio de 17.32 meses. Las madres se encontraban casadas, con 2 hijos o más, $87 \%$ vivía con 4 o más personas en su domicilio. El 91\% de las madres entrevistadas vivía en casa propia, el 98\% en casa de loza. $48 \%$ contaba con aparatos electrodomésticos. En 93\% de los hogares 2 personas recibían un sueldo, mayormente trabajando de tiempo completo. En el 56\% de los casos 4 o más personas dependían de los ingresos familiares. La escolaridad moda fue preparatoria. $67 \%$ realizaba labores del hogar, 52\% contaba con Seguro Popular. Las infusiones se introdujeron al tercer mes

a Estudiante de Maestría en Seguridad Alimentaria y Nutricional de la Universidad Veracruzana. https://orcid.org/0000-0003-0069-6637, Email: zS20000682@estudiantes.uv.mx

b Autor de correspondencia. Centro de Investigación en Nutrición y Alimentación de la Licenciatura en Nutrición. Universidad del Istmo. https://orcid.org/0000-0002-6504-7366, Email: eoi@bizendaa.unistmo.edu.mx

• Centro de Investigación en Nutrición y Alimentación de la Licenciatura en Nutrición. Universidad del Istmo. https://orcid.org/0000-00021104-2949, Email: ihoi@bizendaa.unistmo.edu.mx 
como primera bebida diferente a la leche materna. $98 \%$ poseían una diversidad alimentaria alta, el grupo de mayor consumo en el hogar familiar fue cereales. Conclusiones: Observación sociodemográfica a 46 madres que en su mayoría se dedicaban a labores del hogar, contaban con casa propia de concreto, 2 o más hijos y con Seguro popular. Las personas entrevistadas contaban con Seguridad Alimentaria, una moda de inicio de Alimentación Complementaria a los 3 meses con infusiones caseras y las familias contaban con Diversidad Alimentaria Alta.

\section{Palabras Clave:}

Lactancia materna, Alimentación complementaria, Seguridad Alimentaria, Diversidad Alimentaria

\section{Introducción}

La nutrición desde etapas tempranas de la vida impacta en el crecimiento y constituye un factor de riesgo para enfermedades crónicas futuras. La leche humana es el alimento diseñado para brindar al lactante información inmunológica y nutricional. La Alimentación Complementaria (AC) abarca de los 6 meses a los 2 años de edad periodo en el cual la leche de la madre deja de ser suficiente para cubrir las necesidades nutricionales del niño y por lo tanto, se deben agregar otros alimentos de forma complementaria a su dieta, es decir, la madre brinda Lactancia Materna Complementaria a los alimentos sólidos del bebé. .,9-11 $^{-1}$

La producción y la composición de la leche se relacionan con tres aspectos de la nutrición materna: la alimentación habitual, reservas de nutrientes y energía y las alteraciones en la utilización de las nutrientes influenciadas por el entorno hormonal que caracteriza al período. $^{1}$

La nutrición de las madres y sus hijos es una de las herramientas más efectivas para lograr el óptimo crecimiento y desarrollo del ser humano. ${ }^{2}$ Se dice que "un hogar tiene Seguridad Alimentaria cuando tiene acceso a la alimentación requerida para una vida saludable para todos sus miembros (adecuada en términos de calidad, cantidad y aceptable culturalmente) y cuando no está en riesgo de perder dicho acceso".,4 Por lo tanto, la Inseguridad Alimentaria se ve como la combinación de dos problemas: el acceso y el uso de los alimentos. ${ }^{8}$

El objetivo del presente fue establecer el Nivel de Seguridad Alimentaria y Nutricional de las madres que brindan Lactancia Materna Complementaria a bebés de 6 a 24 meses de edad, mediante un estudio descriptivo, observacional, transversal y retrospectivo con el empleo de encuestas validadas, realizadas individual a las madres en sus domicilios.

\section{Método}

Se realizó un estudio de diseño descriptivo, transversal y retrospectivo, Los instrumentos de medición fueron Escala Mexicana de Seguridad Alimentaria (EMSA). México, ENIGH 2008; para medir la Inseguridad Alimentaria y variedad de la alimentación en hogares mexicanos con niños menores de cinco años. La Encuesta sociodemográfica (Basada en encuesta Sociodemográfica CUNE), así como la Encuesta Introducción de Alimentación Complementaria-Propuesta Xunaxi (Adaptada de Cuestionario Lactancia CLAC), para conocer el tipo de alimento y el momento en que se le brindó al niño, determinando así el inicio de la Alimentación Complementaria. Por último, el Cuestionario de Diversidad Dietética en el Hogar para la Medición del Acceso a los Alimentos en el Hogar. La muestra fue asignada a consideración, acudiendo al domicilio de la mayor cantidad de madres que cumplieron con las características de inclusión para el estudio.

Autorizaron las madres mediante el consentimiento informado (obtenido y modificado) del Instituto Nacional de Salud Pública y bajo los criterios de la Declaración de Helsinki (Principios éticos para las investigaciones médicas en seres humanos) que en los principios generales menciona "velar solícitamente y ante todo por la salud de mi paciente". Así mismo se basa en la Ley General de Salud que reglamenta el derecho a la protección de la salud que tiene toda persona en los términos del Artículo 4o. de la Constitución Política de los Estados Unidos Mexicanos. De la misma manera se tomó en cuenta la Norma Oficial Mexicana 012 (NORMA Oficial Mexicana NOM-012-SSA3-2012) que establece los criterios para la ejecución de proyectos de investigación para la salud en seres humanos.

Se describió estadísticamente porcentajes y medidas de tendencia central según correspondió a las variables en estudio. La información se capturó en hojas de cálculo de Microsoft Office Excel 2013, que fungieron como base de datos en la cual se transcribieron las respuestas de entrevistas, con el fin de análisis descriptivo.

\section{Resultados}

De la Escala Mexicana de Seguridad Alimentaria (E.M.S.A.)

Nivel de Seguridad Alimentaria E.M.S.A: Los intervalos para la interpretación de los resultados fueron para Seguridad alimentaria quienes en su entrevista obtuvieron 
0 puntos, Inseguridad leve para quienes tuvieron un resultado de 1-5, Inseguridad moderada para las puntuaciones 6-10 y con Inseguridad severa al tener un resultado de 11-15 puntos. EL $96 \%$ de las personas entrevistadas cuentan con Seguridad Alimentaria y tan solo $4 \%$ varió al resultar con Inseguridad moderada.

Tabla 1. Resultados de la hoja de recolección de datos.

\begin{tabular}{|c|c|c|c|c|}
\hline $\begin{array}{l}\text { Dato en } \\
\text { cuestión }\end{array}$ & Moda & Media & Mínimo & Máximo \\
\hline $\begin{array}{l}\text { Edad de la } \\
\text { madre* }^{*}\end{array}$ & $26^{\mathrm{a}}$ & 29.86 & 20 & 43 \\
\hline $\begin{array}{l}\text { Edad del } \\
\text { bebét }^{* *}\end{array}$ & $24^{b}$ & 17.32 & 6 & 24 \\
\hline
\end{tabular}

*Años cumplidos; aLas madres de los municipios participantes fueron, Ciudad Ixtepec y Magdalena Tlacotepec, este último con la mayor afluencia; " edad $\mathrm{y}$ bebés de ambos géneros, el masculino con mayor representatividad.

Tabla 1. Resultados de la E.M.S.A.

\begin{tabular}{|l|l|}
\hline Dato en cuestión & $\%$ \\
\hline Seguridad Alimentaria & 96 \\
\hline Inseguridad Alimentaria & 4 \\
\hline Total & 100 \\
\hline
\end{tabular}

De la Encuesta Sociodemográfica

Tabla 3. Resultados de la Encuesta Sociodemográfica.

\begin{tabular}{|c|c|c|}
\hline Dato en cuestión & Opción & $\%$ \\
\hline \multirow[t]{5}{*}{ Estado civil } & Casada & 72 \\
\hline & Unión libre & 22 \\
\hline & Divorciado(a) o separados & 2 \\
\hline & Viudo(a) & 0 \\
\hline & Soltero(a) & 4 \\
\hline \multirow[t]{2}{*}{ Número de hijos } & 1 & 24 \\
\hline & 20 más & 76 \\
\hline \multirow{3}{*}{$\begin{array}{c}\text { Número de personas que viven } \\
\text { en su casa }\end{array}$} & 2 & 0 \\
\hline & 3 & 13 \\
\hline & 4 o más & 87 \\
\hline \multirow[t]{2}{*}{ Vivienda } & Propia & 91 \\
\hline & Rentada & 9 \\
\hline \multirow[t]{2}{*}{ Material de la vivienda } & Lámina, cartón, madera & 2 \\
\hline & Loza, concreto & 98 \\
\hline $\begin{array}{l}\text { Aparatos con los que cuenta } \\
\text { para su vida cotidiana }\end{array}$ & $\begin{array}{c}\text { Lavadora, Refrigerador, Computadora, } \\
\text { Televisor, Teléfono }\end{array}$ & 48 \\
\hline
\end{tabular}




\begin{tabular}{|c|c|c|}
\hline & $\begin{array}{c}\text { Lavadora, Refrigerador, Televisor, } \\
\text { Teléfono }\end{array}$ & 20 \\
\hline & Refrigerador, Televisor & 11 \\
\hline & Lavadora, Refrigerador, Televisor & 9 \\
\hline & Refrigerador, & 4 \\
\hline & Computadora, Refrigerador, Televisor & 4 \\
\hline & Lavadora & 2 \\
\hline & $\begin{array}{c}\text { Refrigerador, } \\
\text { Computadora, Televisor, Teléfono }\end{array}$ & 2 \\
\hline \multirow{3}{*}{$\begin{array}{l}\text { Personas en su casa que } \\
\text { trabajan y reciben un salario }\end{array}$} & 2 & 93 \\
\hline & 3 & 7 \\
\hline & 4 o más & 0 \\
\hline \multirow[t]{2}{*}{ Trabajan de tiempo completo } & Si & 65 \\
\hline & No & 35 \\
\hline \multirow{3}{*}{$\begin{array}{l}\text { Número de personas que } \\
\text { dependen de los ingresos de la } \\
\text { familia }\end{array}$} & 2 & 9 \\
\hline & 3 & 35 \\
\hline & 40 más & 56 \\
\hline \multirow{6}{*}{$\begin{array}{c}\text { ¿Qué nivel de escolaridad } \\
\text { posee? }\end{array}$} & Ninguno & 5 \\
\hline & Primaria & 2 \\
\hline & Secundaria & 28 \\
\hline & Preparatoria & 39 \\
\hline & Universidad & 26 \\
\hline & Postgrado & 0 \\
\hline \multirow[t]{2}{*}{ Actividad/empleo que ejerce } & Desempleado & 0 \\
\hline & Labores del hogar & 67 \\
\hline
\end{tabular}




\begin{tabular}{|c|c|c|}
\hline & Comerciante & 24 \\
\hline & Profesionista & 9 \\
\hline Servicio de salud & Agricultura, ganadería o pesca & 0 \\
\hline & Pensionado o jubilado & 0 \\
\hline & Ninguno & 6 \\
\hline IMSS & 15 \\
\hline ISSSTE & 20 \\
\hline & Seguro popular & 52 \\
\hline
\end{tabular}

De la Encuesta de Introducción de Alimentación complementaria

Tabla 2. Moda y promedio de consumo de alimentos al comienzo de la alimentación

\begin{tabular}{|c|c|c|c|}
\hline Tipo de alimento & $\begin{array}{c}\text { Tipo de } \\
\text { preparación }\end{array}$ & $\begin{array}{c}\text { Moda de la edad del niño } \\
\text { (meses) al comienzo del } \\
\text { alimento }\end{array}$ & $\begin{array}{c}\text { Promedio de la frecuencia } \\
\text { (veces/semana) }\end{array}$ \\
\hline Verduras crudas & Casero & 12 & 1 \\
\hline Verduras cocidas & Casero & 6 & 4 \\
\hline Frutas & Casero & 6 & 4 \\
\hline Jugo de frutas & Casero & 6 y 10 & 6 \\
\hline Cereales & Casero & 6 & 2 \\
\hline Carne de pollo & Casero & 8 y 12 & 1 \\
\hline Carne de res & Casero & 12 & 1 \\
\hline Pescado & Casero & 12 & 2 \\
\hline Huevo & -- & 12 & 2 \\
\hline Embutidos & -- & 12 & 3 \\
\hline Quesos & Casero & 12 & 5 \\
\hline Yogurt & Marca & 7 & 1 \\
\hline Leche de vaca & Entera & 6 & 1 \\
\hline Chucherías & -- & 12 & 1 \\
\hline Infusiones & Casero & 3 & 12 \\
\hline Refrescos & Diversas marcas & & 12 \\
\hline
\end{tabular}

Se consultaron 16 grupos de alimentos para fines de esta investigación como se muestra en la Tabla 4, se puede observar que las infusiones representan la moda más temprana con 3 meses, con un promedio de frecuencia de consumo de 1 día a la semana.

Los grupos de verduras cocidas, frutas, jugo de frutas, cereales y leche de vaca tuvieron moda de 
introducción de 6 meses de edad con una frecuencia de consumo a la semana de 4 días, 4 días, 4 días, 6 días y 5 días respectivamente.

La introducción a la alimentación del niño con carne de pollo tuvo una moda de 8 meses con una frecuencia de consumo semanal de 2 días.

Las verduras crudas, carne de res, pescado, huevo, embutidos, quesos, chucherías y refrescos tuvieron una moda de 12 meses con frecuencia de 1 día, 1 día, 1 día, 2 días, 1 día, 2 días, 1 día y 1 día respectivamente. La moda de introducción de carne de pollo se repite a los 12 meses con una frecuencia de 2 días a la semana.

Del Cuestionario de Diversidad Dietética en el Hogar para la Medición del Acceso a los Alimentos en el Hogar

Tabla 3. Clasificación de Diversidad Dietética en el Hogar.

\begin{tabular}{|l|l|l|l|}
\hline Clasificación & \multicolumn{2}{l|}{ Total } & $\%$ \\
\hline $\begin{array}{l}\text { 0-3 grupos } \\
\text { alimentos }\end{array}$ & $\begin{array}{l}\text { Diversidad } \\
\text { Alimentaria baja }\end{array}$ & 0 & 0 \\
\hline $\begin{array}{l}\text { 4-5 grupos } \\
\text { alimentos }\end{array}$ & $\begin{array}{l}\text { Diversidad } \\
\text { Alimentaria media }\end{array}$ & 1 & 2 \\
\hline $\begin{array}{l}\text { 6-12 grupos } \\
\text { alimentos }\end{array}$ & $\begin{array}{l}\text { Diversidad } \\
\text { Alimentaria alta }\end{array}$ & 45 & 98 \\
\hline
\end{tabular}

98\% de las madres entrevistadas (45 madres), de acuerdo a sus respuestas y puntajes obtenidos por grupos de alimentos consumidos en el hogar poseen una diversidad alimentaria alta. Tan solo el $2 \%$ posee diversidad alimentaria media.

Tabla 4. Lugar asignado por consumo de 24 horas.

\begin{tabular}{|c|c|c|}
\hline $\begin{array}{l}\text { Lugar } \\
\text { consumo }\end{array}$ & $\begin{array}{l}\text { Tabulaci } \\
\text { ón }\end{array}$ & $\begin{array}{l}\text { Total } \\
\text { hogares }\end{array}$ \\
\hline 1 & Cereales & 44 \\
\hline 2 & Verduras & 43 \\
\hline 3 & Azúcar & 41 \\
\hline 4 & Carne & 40 \\
\hline 5 & Lácteos & 40 \\
\hline 6 & $\begin{array}{l}\text { Condimentos, café, } \\
\text { té }\end{array}$ & 38 \\
\hline 7 & Frijoles, lentejas & 37 \\
\hline 8 & Grasas & 37 \\
\hline 9 & Frutas & 36 \\
\hline 10 & Tubérculos & 19 \\
\hline 11 & Huevo & 19 \\
\hline 12 & Pescado, mariscos & 16 \\
\hline
\end{tabular}

El grupo de alimentos del que mayor registro se tuvo fue cereal con $11 \%$, seguido por verduras (10\%), azúcar (10\%), carne (10\%), lácteos $(10 \%)$, condimentos cafés, té $(9 \%)$, frijoles, lentejas $(9 \%)$, grasas $(9 \%)$, frutas $(9 \%)$, huevo $(5 \%)$, tubérculos $(4 \%)$, pescado, mariscos (4\%).

\section{Discusión}

La edad de los bebés presentó una moda de 24 meses de edad y en su mayoría (54\%) fueron sexo masculino, este último dato es relevante pues según INEGI en 2015 a nivel nacional había 94 hombres por cada 100 mujeres. ${ }^{5}$

De acuerdo a la Escala Mexicana de Seguridad Alimentaria (E.M.S.A.), las respuestas de las madres mostraron que $96 \%$ de los hogares tienen Seguridad Alimentaria y $4 \%$ Inseguridad Alimentaria. En la ENIGH en 2008 Vega M. (et al.) describió que el $52 \%$ de los hogares participantes se encontraba en Seguridad Alimentaria mientras que $48 \%$ en Inseguridad Alimentaria en sus tres grados. ${ }^{6}$ Los resultados de las investigaciones son muy distintos en porcentajes.

De la Encuesta de Introducción de Alimentación Complementaria se obtiene que a partir de un promedio de 3 meses al bebé se le brindó infusiones caseras en frecuencia de 1 día a la semana. Los alimentos que se introdujeron en promedio a los 6 meses de vida fueron verduras cocidas, frutas, jugos de frutas, cereales y leche entera industrializada de vaca. La Organización Mundial de la Salud (OMS) sugiere la introducción de alimentos complementarios a partir de los 6 meses de edad. La misma sugerencia realiza el Instituto Mexicano de Seguro Social (IMSS) para brindar una Lactancia Materna Exclusiva que beneficie el desarrollo óptimo del bebé. ${ }^{7}$

Las costumbres y tradiciones alimentarias son importantes para la determinación de la introducción de alimentos complementarios al bebé.

\section{Conclusiones}

46 madres entrevistadas, las cuales $39 \%$ poseen nivel de escolaridad preparatoria, $67 \%$ realiza labores del hogar y $52 \%$ cuenta con servicio de salud Seguro Popular. 76\% tiene 2 o más hijos, $87 \%$ mencionó que en su vivienda habitan 4 o más personas, $91 \%$ tiene casa propia, $98 \%$ tiene casa de loza, concreto.

La edad promedio de introducción de algún alimento diferente a la leche materna fue de 3 meses y corresponde a infusiones con frecuencia semanal de 1 día.

Según la moda del mes de introducción de los grupos de alimentos, el patrón de introducción de la Alimentación Complementaria fue: 
$98 \%$ de la población entrevistada refirió Diversidad Alimentaria en el Hogar alta.

$96 \%$ de las madres entrevistadas obtuvo la puntuación correspondiente a tener Seguridad Alimentaria.

La capacitación del personal de salud referente a los nuevos conocimientos de patrón de introducción de la alimentación complementaria beneficiará a madres y sus bebés para lograr una mejor salud.

La iniciativa de grupos de apoyo entre madres con hijos en la misma etapa o similar bajo la supervisión de un especialista podrá brindar seguridad a la hora de brindar nuevos alimentos o preparaciones a la familia.

La orientación a las madres acerca del empleo de alimentos locales ricos en nutrientes para la preparación de sus alimentos beneficiará a la diversidad de grupos de alimentos consumidos en el hogar sin descuidar la Seguridad Alimentaria.

Tabla 5. Patrón de introducción de la Alimentación Complementaria, según la moda observada.

\begin{tabular}{|c|c|}
\hline Edad en meses & Alimento \\
\hline 3 meses de vida & Infusiones \\
\hline 6 meses de vida & $\begin{array}{l}\text { Verduras cocidas, frutas, } \\
\text { jugo de frutas, cereales, } \\
\text { leche de vaca }\end{array}$ \\
\hline 7 meses de vida & Yogurt \\
\hline 8 meses de vida & Carne de pollo \\
\hline 12 meses de vida & $\begin{array}{l}\text { Verduras crudas, carne de } \\
\text { res, pescado, huevo, } \\
\text { embutidos, quesos, } \\
\text { chucherías y refrescos }\end{array}$ \\
\hline
\end{tabular}

Financiamiento: ninguno

Conflicto de intereses: ninguno

\section{Referencias}

[1] Olagnero G, Barreto L, Terraza R, Wiedemann A, Poy M, López L. Alimentación de la mujer en período de lactancia. Actualización en nutrición. Argentina. 2017: 99-105.

[2] Vásquez E. Primer año de vida. Leche humana y sucedáneos de la leche humana. Gac Med Mex;152 Suppl 1:13-21; 2016.
[3] Secretaría de Desarrollo Social. Nutrición y pobreza: política pública basada en evidencia. México; 2008.

[4] Barrientos, M, Flores S. ¿Es la obesidad un problema médico individual y social? Políticas públicas que se requieren para su prevención, Boletín Médico del Hospital Infantil de México, vol. 65 noviembre-diciembre; 2008.

[5] INEGI. Población mujeres y hombres en México (sitio en internet). Disponible en http://cuentame.inegi.org.mx/poblacion/mujeresyhombres.aspx?tema $=\mathrm{P}$

[6] Instituto Mexicano del Seguro Social. Lactancia. IMSS. México; 2017. Disponible en: http://www.imss.gob.mx/salud-enlinea/lactancia

[7] Vega M, Levy T, Peinador R, Méndez I, Melgar H. Inseguridad alimentaria y variedad de la alimentación en hogares mexicanos con niños menores de cinco años. Salud pública Méx vol.56 supl.1. Cuernavaca; 2014

[8] Consejo Nacional de Evaluación de la Política de Desarrollo Social Dimensiones de la seguridad alimentaria: Evaluación Estratégica de Nutrición y Abasto. CONEVAL, México; 2010.

[9] Organización Mundial de la Salud. Alimentación Complementaria Temas de Nutrición. 2016.

[10] Comité de Lactancia Materna · Asociación Española de Pediatría. Recomendaciones sobre lactancia materna. AEP-CLM. España; 2012.

[11] Martínez R. et al. Estudio de las pautas alimentarias para la introducción de alimentos complementarios y su diversidad a través de la incorporación a la dieta familiar. Revista de Especialidades Médico-Quirúrgicas;15(3):114-124. México; 2010. 